\title{
High tumor mutation burden in a patient with metastatic gastric cancer sensitive to trastuzumab: a case report
}

\author{
Chun-Ting $\mathrm{Hu}^{1}$, Yi-Chen Zhou ${ }^{1}$, Li-Dong $\mathrm{Zu}^{1}$, Guo-Hui Fu' ${ }^{1}$, Qi Li $^{2}$ \\ ${ }^{1}$ Pathology Center, Shanghai General Hospital/Faculty of Basic Medicine, Key Laboratory of Cell Differentiation and Apoptosis of Chinese Ministry \\ of Education, Institutes of Medical Sciences, Shanghai Jiao Tong University School of Medicine, Shanghai, China; ${ }^{2}$ Department of Oncology, \\ Shanghai General Hospital, Shanghai Jiaotong University, Shanghai, China \\ Correspondence to: Guo-Hui Fu. Pathology Center, Shanghai General Hospital/Faculty of Basic Medicine, Key Laboratory of Cell Differentiation and \\ Apoptosis of Chinese Ministry of Education, Institutes of Medical Sciences, Shanghai Jiao Tong University School of Medicine, Shanghai, China. \\ Email: guohuifu@shsmu.edu.cn; Qi Li. Department of Oncology, Shanghai General Hospital, Shanghai Jiaotong University, Shanghai, China. \\ Email: leeqi2001@hotmail.com.
}

\begin{abstract}
Patients with HER2-positive gastric cancer (GC) can benefit from the addition of trastuzumab. However, not all patients with HER2-positive GC respond to trastuzumab. Biomarkers affecting its efficacy in patients with advanced gastric cancer (AGC) are largely unknown. Therefore, classifying GC into molecularly distinct subtypes to accurately distinguish between GC patients who would and would not benefit from trastuzumab is worthwhile. Tumor mutation burden (TMB) is a notable feature in GC and whether TMB influences trastuzumab efficacy is still unknown. Herein, we report the case of a 61-yearold man who was diagnosed with metastatic HER2-positive gastric adenocarcinoma that had spread to the liver (T4aN0M1, stage IV). Esophagogastroduodenoscopy revealed a circular ulcer in the posterior wall of the stomach. A computed tomography (CT) scan revealed a 2-cm diameter liver metastasis. Immunohistochemical analysis of the endoscopic biopsy tumor revealed 3+positive expression for HER2. Whole-exome sequencing (WES) of the tumor tissue revealed 3,736 somatic mutations in 2,423 genes and a very high TMB of 50.3 mutations/Mb. Immunohistochemistry revealed that the patient had mismatch repair-proficient (pMMR) GC. The patient received first-line trastuzumab-containing chemotherapy, and after 2 courses of sequential metronomic trastuzumab-containing chemotherapy, restaging CT showed that the liver metastasis had disappeared. Following resection, the patient had no recurrence and no new tumor metastasis after a follow-up of period nearly 7 years. This study is the first to report that pMMR GC with a high TMB has a favorable response to trastuzumab. The combination of HER2 positivity and a high TMB may be sufficiently predictive of sensitivity to trastuzumab in AGC.
\end{abstract}

Keywords: Gastric cancer (GC); HER2; trastuzumab; tumor mutation burden (TMB); targeted therapy; case report

Submitted Jan 08, 2020. Accepted for publication Aug 18, 2020.

doi: $10.21037 /$ apm-20-132

View this article at: http://dx.doi.org/10.21037/apm-20-132

\section{Introduction}

Approximately 20\% of gastric cancers (GCs) overexpress the HER2 receptor (1-3). Trastuzumab is a HER2-targeted humanized monoclonal antibody that has been studied as a first-line treatment for patients with HER2-positive advanced gastric cancer (AGC). ToGA (Trastuzumab for
Gastric Cancer) revealed a significant overall survival benefit in patients with HER2-positive AGC (2), but the overall response rate was $47 \%$, and the complete response rate was only $5 \%$. Therefore, only a minority of patients derive lasting benefits from trastuzumab therapy. Moreover, the available data clearly demonstrate that high-dose (HD) trastuzumab maintenance dosing is not associated with 
increased efficacy (4). Therefore, potential biomarkers for high response rates to anti-HER2 therapies still need to be identified. Tumor mutation burden (TMB) may predict the clinical response to immune checkpoint inhibitors (ICIs) (5). A high TMB is associated with improved immunotherapy outcomes in patients with esophagogastric cancer, with those with a TMB $>9.7$ mutations (muts)/Mb (in the top quartile) having the best outcomes (6). However, the role of TMB in targeted therapy in patients based on their response to trastuzumab has not been reported.

Herein, we report a significant elevation in TMB in a liver metastatic GC patient who was highly responsive to trastuzumab. As TMB is a recognized predictive biomarker for the response to ICIs in esophagogastric cancer, we speculated that patients with HER2-positive tumors with a high TMB might receive more benefit from trastuzumab than those with a low TMB. We present the following article/case in accordance with the CARE Reporting Checklist (available at http://dx.doi.org/10.21037/apm-20132).

\section{Case presentation}

A 61-year-old man with a BMI of 24.2 presented with mild discomfort under the xiphoid process from the age of 58 [2009] without apparent cause. He was admitted to our hospital with recurrent increasing abdominal distension for 2 months in October 2012. The patient reported that the distension pain occurred two or three hours after a meal and was accompanied by nausea and vomiting. The patient had no significant medical history, and there was no apparent weight loss or pain in either his abdominal or back and flank pain at that time; thus, the patient did not pay much attention to the condition. During the physical examination, it was noted that the upper abdomen was mildly tender, with no mass felt in the upper abdomen and no swelling upon palpating the superficial lymph nodes (LNs) throughout the body. The liver and could not be palpated under the ribs. Laboratory examinations revealed a low hemoglobin concentration of $108 \mathrm{~g} / \mathrm{L}$, carcinoembryonic antigen (CEA) $(4.04 \mathrm{ng} / \mathrm{mL})$, alpha-fetoprotein (AFP) $(2.94 \mathrm{ng} / \mathrm{mL})$ and carbohydrate antigen 19-9 (CA19-9) (19.22 U/mL) levels were normal. Esophagogastroduodenoscopy revealed a $30-\mathrm{mm}$ circular ulcer in the posterior wall of the lower stomach body. Endoscopic biopsy specimens were obtained, and the pathology of the specimens revealed moderately differentiated gastric adenocarcinoma. Immunohistochemistry was strongly positive for HER2 receptor expression (3+). A computed tomography (CT) scan revealed a thickening of the lower part of the stomach and the wall of the antrum and hepatogastric ligament and swollen LNs near the abdominal aorta, with a metastatic lesion $2 \mathrm{~cm}$ in diameter in the lower right lobe of the liver. He was diagnosed with liver metastatic GC in December 2012. The tumor was classified as cT4aN0M1, stage IV.

Based on the strong positivity for HER2 (Figure 1A), trastuzumab-based XELOX chemotherapy [consisting of a 2-h i.v. infusion of $130 \mathrm{mg} / \mathrm{m}^{2}$ oxaliplatin on day 1 and $1,000 \mathrm{mg} / \mathrm{m}^{2}$ oral capecitabine twice daily on days $1-15$, plus $8 \mathrm{mg} / \mathrm{kg}$ trastuzumab on day 1 (second course $6 \mathrm{mg} / \mathrm{kg}$ )] was administered every 3 weeks from December 14 , 2012, and repeated for 12 courses. After 2 trastuzumabbased chemotherapy courses, restaging CT showed that the liver metastasis had disappeared on February 2, 2012, and that the size of the primary tumor in the stomach was similar to before. CT scans showed partial response (PR) in gastric and liver metastases by RECIST version 1.1. The patient tolerated trastuzumab treatment well, without severe adverse events. Serum tumor marker levels were evaluated, including CEA $(3.04 \mathrm{ng} / \mathrm{mL})$, AFP $(1.64 \mathrm{ng} / \mathrm{mL})$ and CA19-9 $(8.82 \mathrm{U} / \mathrm{mL})$. After 12 courses, the patient was confirmed to have stable disease (SD) by CT. Primary tumor changes were not obvious, therefore, the patient underwent radical gastric resection on November 2013; a persistent histopathological diagnosis was made of moderately differentiated adenocarcinoma, tumor cell expansive invasion to the peritoneal layer of the stomach, and 27 LNs without metastasis. The patient was classified as having stage IV (pT4aN0M1) disease. Nevertheless, HER2 immunohistochemistry in the surgical biopsy demonstrated the loss of HER2 positivity. After surgery, the patient was placed on a regimen of tegafur-uracil $(120 \mathrm{mg}$ per day orally) for sixteen months. The patient tolerated treatment with trastuzumab and chemotherapy well. Except for mild nausea and vomiting, which subsided after symptomatic treatment, no additional adverse events were observed. The patient returned for regular follow-up visits at the hospital without adjuvant treatment (see treatment timeline, Figure $1 B$ ). Follow-up visits in the outpatient department every 3-6 months. Laboratory data indicated normal levels of tumor markers, and subsequent monitoring by CT and MRI showed no regional LN swelling, no ascites and multiple metastatic tumors. In addition, no adverse event was observed in the follow up period. As of November 2019, the patient did not have any recurrence or new metastases for 72 months. 
A

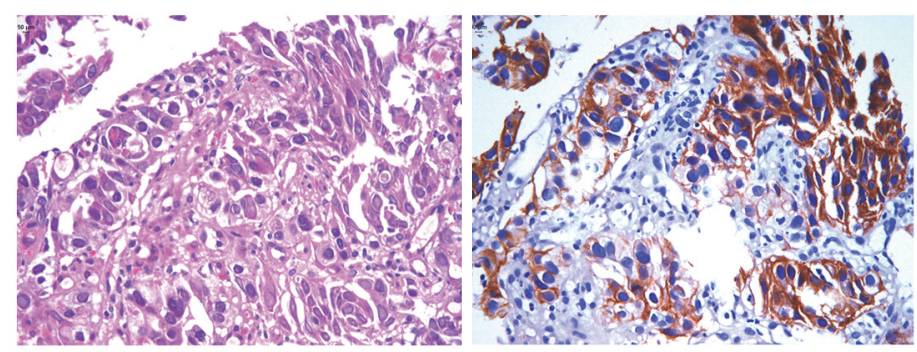

B

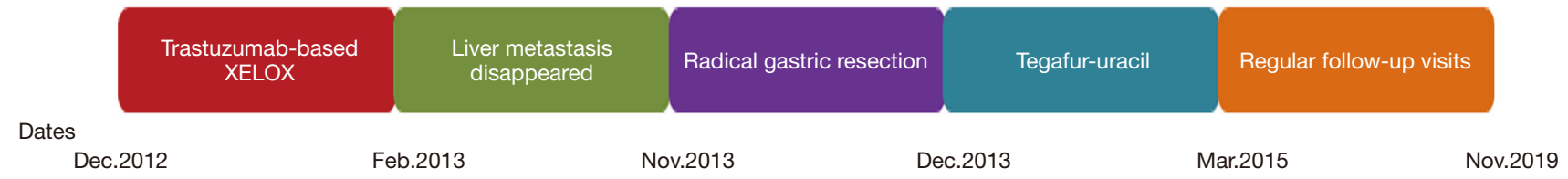

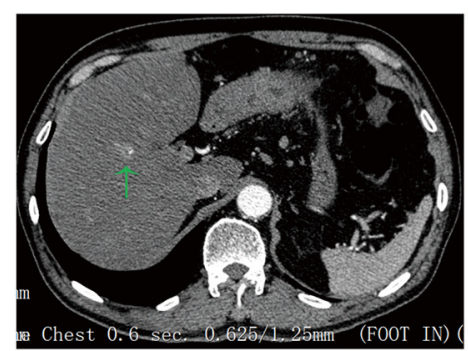

Before trasuzumab

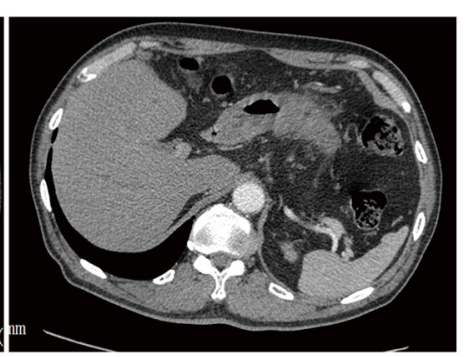

Response to trasuzumab

C

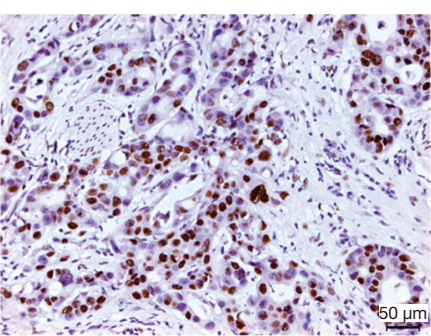

MLH1

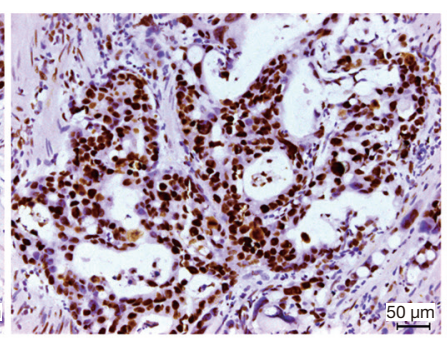

MHS2

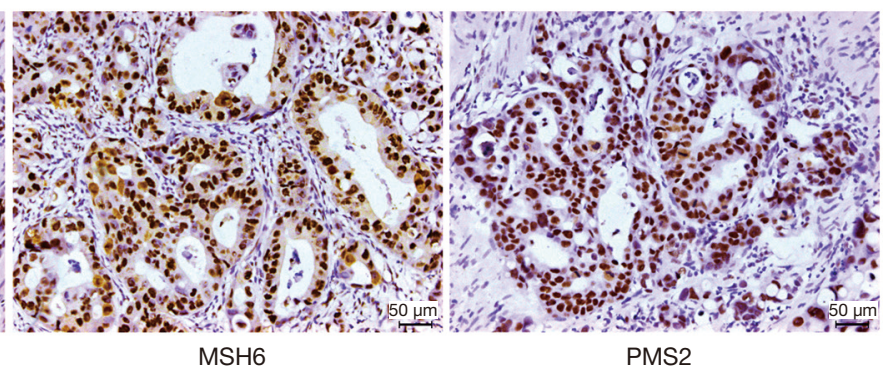

PMS2

Figure 1 HER2 immunohistochemistry and therapy timeline. (A) Left, histopathology of the GC specimens obtained from the endoscopic biopsy, H\&E stain; right, immunohistochemical positivity for the HER2 protein. Original magnification $\times 400$. (B) Therapy timeline; CT image showing a complete response in the liver metastasis (green arrow) of this patient after 2 courses of trastuzumab treatment. (C) Representative immunohistochemistry images of GC samples showing strong nuclear expression of MMR proteins (immunohistochemistry $\times 100$, the scale bar indicates $50 \mu \mathrm{m})$. GC, gastric cancer; CT, computed tomography; MMR, mismatch repair.

We collected formalin-fixed paraffin-embedded (FFPE) tissue slides containing gastric carcinoma tissue and paired noncarcinoma tissue for whole-exome sequencing (WES) with the Illumina NovaSeq system. We obtained 70-fold mean sequence coverage of the targeted exonic regions, with $97.53 \%$ of loci covering $>10$-fold sequences. To confirm the somatic (i.e., tumor-specific) nature of these mutations, we compared these data with those from the matched normal tissue. We identified a total of 3,736 putative somatic point mutations in 2,423 genes. The somatic mutation types and their prevalence in the patient are shown in Table 1. Several mutations were located in nine highly variable genes: AHNAK2, MUC4, SULT1A1, NBPF1, ABO, MST1L, ZNF718, MUC12 and MUC16 (Table S1). In particular, a very high TMB was observed in this patient (50.3 muts/Mb). Notably, WES revealed a lack of mutations in mismatch 
Table 1 Summary of somatic mutation types and prevalence in patient

\begin{tabular}{lc}
\hline Mutation types & Prevalence \\
\hline Coding regions and essential splice sites & \\
SNV & 755 \\
Synonymous & \\
Nonsynonymous & 839 \\
Missense & 21 \\
Splicing & 17 \\
Stopgain & 0 \\
Stoploss & \\
Non-coding regions & 78 \\
Indel & 1,633 \\
SNV & 393 \\
Indel & \\
\hline
\end{tabular}

SNV, single-nucleotide variations.

repair (MMR) genes (MLH1, MSH2, PMS2 and MSH6), and following immunohistochemistry, the MMR proteins retained nuclear staining and were deemed mismatch-repair proficient (Figure 1C).

All procedures performed in studies involving human participants were in accordance with the ethical standards of the institutional and/or national research committee(s) and with the Helsinki Declaration (as revised in 2013). The patient of this case report agreed and signed consent forms, which are attached to the medical records. Written informed consent was for the purpose of publication of the present case report and any relevant images.

\section{Discussion}

GC is a biologically heterogeneous tumor (7). The characterization of GC into subtypes based on genotype has evolved over the past decade (8). The Cancer Genome Atlas (TCGA) categorized 295 GC samples into four key subtypes, namely, Epstein-Barr virus (EBV)positive, microsatellite instability (MSI), genomically stable (GS) and chromosomal instability (CIN) (9). Similarly, the Asian Cancer Research Group (ACRG) analyzed 300 primary GC samples and described four molecular subtypes linked to distinct clinical outcomes and prognoses, namely, MSI, microsatellite stable (MSS) mesenchymal-like type tumors, and TP53-active
(MSS/TP53+) and TP53-inactive (MSS/TP53-) (10); furthermore, they differentiated between two distinct molecular subtypes, namely, the mesenchymal phenotype (MP) and the epithelial phenotype (EP). The MP subtype is associated with markedly poor survival and resistance to standard chemotherapy (7). However, multiplex subtypes cannot serve as biomarkers to evaluate the treatment response to immunotherapy or to aid in decisions regarding targeted therapy.

TMB, as measured by WES, has been shown to strongly correlate with objective responses to ICIs in several tumor types $(5,11,12)$, and research has validated the role of TMB as a biomarker for patient selection for immunotherapy $(13,14)$. However, whether TMB is related to the appropriate selection of targeted therapy remains unclear. Moreover, the TMB cutoff points associated with improved survival vary markedly among cancer types. In esophagogastric cancer patients, the TMB cutoff point for the top $20 \%$ was approximately $8.8 / \mathrm{Mb}$, although the effect did not reach statistical significance, possibly because of the small sample size, and relevant numerical data have been reported only once (5). Janjigian et al. also revealed that three patients with long immunotherapy responses had a high TMB (59.4, 28.3, and $14.2 \mathrm{muts} / \mathrm{Mb}$ ), and these patients remained alive for 19.5 to 44.7 months following the initiation of immunotherapy despite prior rapid progression while receiving standard chemotherapies (6). In our case report, the TMB of the patient was 50.3 muts $/ \mathrm{Mb}$, which is much higher than the cutoff value used to define the top $20 \%$, but the MMR proteins were mismatch-repair proficient. Trastuzumab is assumed to prime antibody-dependent cellular cytotoxicity (ADCC) (15) and is the main antitumor mechanism of trastuzumab (16). Samples with a high TMB have been associated with increased activated natural killer (NK) cells in non-small-cell lung cancer (17). Our findings suggest that a high TMB activates NK cell-mediated GC cell lysis, such as ADCC, which is associated with a favorable response to trastuzumab in the treatment of GC. This study provided the first data on the effectiveness of trastuzumab therapy in a late-stage GC patient with high TMB, pMMR and positive HER2 expression. However, due to the ethical issues with performing a liver biopsy, it has not been possible to compare the molecular pattern between primary tumor and liver metastasis. Moreover, whether GC patient with high TMB and negative HER2 expression would have a favorable response to trastuzumab and whether GC patient with high TMB and mismatch repair-deficient 
(dMMR) GC would experience more benefit than those with $\mathrm{pMMR}$ remains to be explored.

Targeting HER2 with trastuzumab is associated with prolonged overall survival and progression-free survival in individuals with HER2-positive AGC (2) and remains an important strategy for the treatment of patients with HER2-positive, metastatic or AGC. However, the value of trastuzumab combined with chemotherapy has rarely been investigated in the perioperative setting. A recent trial reported that a histopathological complete response was achieved in $8.3 \%$ and $22.2 \%$ of patients with stomach and esophagogastric junction adenocarcinoma, respectively $(18,19)$. Cunningham et al. reported that the 5-year survival rate of patients with metastatic GC was approximately $20 \%$, which was less than that for patients who underwent perioperative chemotherapy (36\%) (20). The HER2-positive patient with a high TMB described in this report received 12 courses of trastuzumab-based XELOX chemotherapy, and his liver metastasis completely disappeared, providing him with the chance to undergo radical resection; this patient showed a favorable course of disease. His general condition was stable, without comorbidities, and remains alive today. Thus, TMB might be an important biomarker for perioperative targeted therapy.

Recently, studies have suggested that chemotherapy, especially trastuzumab treatment, is associated with a significant loss of HER2 overexpression in breast cancer (21) and GC $(22,23)$. Although these studies suggest the need to reexamine the HER2 status before initiating secondline anti-HER2 treatment, there is disagreement on the prognostic role of the loss of HER2 positivity in patients receiving first-line trastuzumab-based treatment $(22,24,25)$. Therefore, the loss of HER2 expression is not well established as a valid indicator of trastuzumab treatment.

\section{Conclusions}

In this case, we report an elderly man with HER2positive liver metastatic $\mathrm{pMMR}$ gastric adenocarcinoma who had a very high TMB and a strong response to trastuzumab therapy, with nearly complete disappearance of the metastasis, providing the patient with the chance to undergo primary lesion resection and significantly prolonging his survival time. We suggest that considering the patient's TMB status can lead to a more accurate prediction of the response to trastuzumab than considering the patient's HER2 status, and trastuzumab therapy should be considered for patients who were previously regarded as unsuitable. Our study provides a new option for maximizing the benefit of trastuzumab therapy in late-stage GC patients. The determination of TMB can allow for a precise identification of the patient population who can potentially benefit from targeted therapy with trastuzumab.

\section{Acknowledgments}

Funding: This work was supported by the National Natural Science Foundation of China (NO81372637, NO81602096), National Basic Research Program of China (973 Program) (NO2013CB910903), National Key Technology R\&D Program of China (NO2014BAI09B03) and Key Projects in Shanghai Science \& Technology Pillar Program for Biomedicine (NO14431904700), First Round of 3-year Action Plan to promote clinical skills and clinical innovation in Municipal Hospitals of Shanghai (16CR2039B), Shanghai hospital development center emerging advanced technology joint research project (SHDC12014105).

\section{Footnote}

Reporting Checklist: The authors have completed the CARE Reporting Checklist. Available at http://dx.doi. org/10.21037/apm-20-132

Conflicts of Interest: All authors have completed the ICMJE uniform disclosure form (available at http://dx.doi. org/10.21037/apm-20-132). The authors have no conflicts of interest to declare.

Ethical Statement: The authors are accountable for all aspects of the work in ensuring that questions related to the accuracy or integrity of any part of the work are appropriately investigated and resolved. All procedures performed in studies involving human participants were in accordance with the ethical standards of the institutional and/or national research committee(s) and with the Helsinki Declaration (as revised in 2013). The patient of this case report agreed and signed consent forms, which are attached to the medical records. Written informed consent was for the purpose of publication of the present case report and any relevant images.

Open Access Statement: This is an Open Access article distributed in accordance with the Creative Commons 
Attribution-NonCommercial-NoDerivs 4.0 International License (CC BY-NC-ND 4.0), which permits the noncommercial replication and distribution of the article with the strict proviso that no changes or edits are made and the original work is properly cited (including links to both the formal publication through the relevant DOI and the license). See: https://creativecommons.org/licenses/by-nc-nd/4.0/.

\section{References}

1. Park DI, Yun JW, Park JH, et al. HER-2/neu amplification is an independent prognostic factor in gastric cancer. Dig Dis Sci 2006;51:1371-9.

2. Bang YJ, Van Cutsem E, Feyereislova A, et al. Trastuzumab in combination with chemotherapy versus chemotherapy alone for treatment of HER2-positive advanced gastric or gastro-oesophageal junction cancer (TOGA): a phase 3, open-label, randomised controlled trial. Lancet 2010;376:687-97.

3. Begnami MD, Fukuda E, Fregnani JHTG, et al. Prognostic Implications of Altered Human Epidermal Growth Factor Receptors (HERs) in Gastric Carcinomas: HER2 and HER3 Are Predictors of Poor Outcome. J Clin Oncol 2011;29:3030-6.

4. Shah MA, Xu RH, Bang YJ, et al. HELOISE: Phase IIIb Randomized Multicenter Study Comparing Standard-ofCare and Higher-Dose Trastuzumab Regimens Combined With Chemotherapy as First-Line Therapy in Patients With Human Epidermal Growth Factor Receptor 2-Positive Metastatic Gastric or Gastroesophageal Junction Adenocarcinoma. J Clin Oncol 2017;35:2558-67.

5. Samstein RM, Lee CH, Shoushtari AN, et al. Tumor mutational load predicts survival after immunotherapy across multiple cancer types. Nat Genet 2019;51:202-6.

6. Janjigian YY, Sanchez-Vega F, Jonsson P, et al. Genetic Predictors of Response to Systemic Therapy in Esophagogastric Cancer. Cancer Discov 2018;8:49-58.

7. Oh SC, Sohn BH, Cheong JH, et al. Clinical and genomic landscape of gastric cancer with a mesenchymal phenotype. Nat Commun 2018;9:1777.

8. Lordick F, Janjigian YY. Clinical impact of tumour biology in the management of gastroesophageal cancer. Nat Rev Clin Oncol 2016;13:348-60.

9. Comprehensive molecular characterization of gastric adenocarcinoma. Nature 2014;513:202-9.

10. Cristescu R, Lee J, Nebozhyn M, et al. Molecular analysis of gastric cancer identifies subtypes associated with distinct clinical outcomes. Nat Med 2015;21:449-56.
11. Hellmann MD, Callahan MK, Awad MM, et al. Tumor Mutational Burden and Efficacy of Nivolumab Monotherapy and in Combination with Ipilimumab in Small-Cell Lung Cancer (vol 33, pg 853, 2018). Cancer Cell 2019;35:329.

12. Turajlic S, Litchfield $\mathrm{K}, \mathrm{Xu} \mathrm{H}$, et al. Insertion-anddeletion-derived tumour-specific neoantigens and the immunogenic phenotype: a pan-cancer analysis. Lancet Oncol 2017;18:1009-21.

13. Hellmann MD, Ciuleanu TE, Pluzanski A, et al. Nivolumab plus Ipilimumab in Lung Cancer with a High Tumor Mutational Burden. New Engl J Med 2018;378:2093-104.

14. Goyal G, Lau D, Nagle AM, et al. Tumor mutational burden and other predictive immunotherapy markers in histiocytic neoplasms. Blood 2019;133:1607-10.

15. Collins DM, O'Donovan N, McGowan PM, et al. Trastuzumab induces antibody-dependent cell-mediated cytotoxicity (ADCC) in HER-2-non-amplified breast cancer cell lines. Ann Oncol 2012;23:1788-95.

16. Moasser MM. Two dimensions in targeting HER2. J Clin Oncol 2014;32:2074-7.

17. Chen $\mathrm{H}$, Chong $\mathrm{W}$, Teng $\mathrm{C}$, et al. The immune responserelated mutational signatures and driver genes in nonsmall-cell lung cancer. Cancer Sci 2019;110:2348-56.

18. Rivera F, Jimenez-Fonseca P, Alfonso PG, et al. NEOHX study: Perioperative treatment with trastuzumab in combination with capecitabine and oxaliplatin (XELOX-T) in patients with HER-2 resectable stomach or esophagogastric junction (EGJ) adenocarcinoma-18 m DFS analysis. J Clin Oncol 2015;33:abstr 107.

19. Hofheinz R, Hegewisch-Becker S, Thuss-Patience PC, et al. HER-FLOT: Trastuzumab in combination with FLOT as perioperative treatment for patients with HER2-positive locally advanced esophagogastric adenocarcinoma: A phase II trial of the AIO Gastric Cancer Study Group. J Clin Oncol 2014;32:abstr 4073.

20. Cunningham D, Allum WH, Stenning SP, et al. Perioperative chemotherapy versus surgery alone for resectable gastroesophageal cancer. New Engl J Med 2006;355:11-20.

21. Ignatov T, Gorbunow F, Eggemann H, et al. Loss of HER2 after HER2-targeted treatment. Breast Cancer Res Treat 2019;175:401-8.

22. Seo S, Ryu MH, Park YS, et al. Loss of HER2 positivity after anti-HER2 chemotherapy in HER2-positive gastric cancer patients: results of the GASTric cancer HER2 reassessment study 3 (GASTHER3). Gastric Cancer 
2019;22:527-35.

23. Pietrantonio F, Caporale M, Morano F, et al. HER2 loss in HER2-positive gastric or gastroesophageal cancer after trastuzumab therapy: Implication for further clinical research. Int J Cancer 2016;139:2859-64.

24. Mittendorf EA, Wu Y, Scaltriti M, et al. Loss of HER2 amplification following trastuzumab-based neoadjuvant

Cite this article as: $\mathrm{Hu}$ CT, Zhou YC, Zu LD, Fu GH, Li Q. High tumor mutation burden in a patient with metastatic gastric cancer sensitive to trastuzumab: a case report. Ann Palliat Med 2021;10(5):5846-5852. doi: 10.21037/apm-20-132 systemic therapy and survival outcomes. Clin Cancer Res 2009; 15:7381-8.

25. Ikari N, Nakajima G, Taniguchi K, et al. HER2-positive gastric cancer with paraaortic nodal metastasis successfully resected after chemotherapy with trastuzumab: a case report. Anticancer Res 2014;34:867-72. 


\begin{tabular}{|c|c|c|c|c|c|c|c|c|c|c|c|}
\hline Genen name & Chromosome & Position & dbSNP ID & REF & ALT & Exonicfunc & Gene & AAChange & cytoBand & cosmic82 & SIFT \\
\hline AHNAK2 & 14 & 105411781 & rs10438247 & G & A & Missense SNV & NM_138420 & AHNAK2:NM_138420:exon7:C.C10007T:P.P3336L & 14432.33 & ID=COSM432734;0CCURENCE=1(thyroid) & $0.014, \mathrm{D}$ \\
\hline AHNAK2 & 14 & 105413223 & rs55797226 & A & $\mathrm{G}$ & Synonymous SNV & NM_138420 & AHNAK2:NM_138420:8xon7:C.T8565C;:D.D2855D & 14432.33 & NA & $\mathrm{NA}$ \\
\hline AHNAK2 & 14 & 1054148810 & 0145032 & c & a & Synonymous SNV & $N M_{1} 138420$ & AHNAK2:NM__138420::xon77:CGG6978C:P..L2326L & 14932.33 & NA & NA \\
\hline AHNAK2 & 14 & 105414923 & 0761412 & T & c & Missense SNV & $N M_{1} 138420$ & AHNAK2:NM__38420::xon77:CA.A6865G:p.:.K2289E & 14932.33 & ID=COSM5433893;:OCCURENCE=1(10esophagus) & $0.148, \mathrm{~T}$ \\
\hline AHNAK2 2 & 14 & 105417588 & rs557610829 & G & A & Synonymous SNV & $N M_{1} 138420$ & AHNAK2:NM_138420::xon77:C.C4200T:P.P1400P & 14932.33 & ID=COSM4147900; OCCURENCE=1(thyroid) & NA \\
\hline AHNAK2 & 14 & 105417597 & rs572861020 & T & c & Synonymous SNV & NM_138420 & HNAK2:NM_138420::Xxon7:AA4191G:P.PP1397P & 14932.33 & ID=COSM4147907;0CCURENCE=1(thyroid) & NA \\
\hline AHNAK2 & 14 & 105417598 & rs201134968 & G & A & Missense SNV & NM_138420 & INAK2:NM_138420::Xxon7:C.C4190T:P.PP1397L & 14432.33 & ID=COSM4147900; OCCURENCE=1(thyroid) & \\
\hline AHNAK2 & 14 & 05417837 & 76231332 & A & G & Synonymous SNV & NM_138420 & HNAK2:NM_138420::xon7:C.T3951C:PAA1377A & 14932.33 & NA & \\
\hline AHNAK2 & 14 & 105418264 & rs55650155 & G & A & Missense SNV & NM_138420 & AHNAK2:NM_138420::8xon7:C.C3524T:PA175V & 14932.33 & $\begin{array}{l}\text { ID=COSM4149917:OCCURENCE=1(thyroid), 1(large_- } \\
\text { intestine), (liver) }\end{array}$ & 1.0,T \\
\hline AHNAK2 & 14 & 105418344 & rs55791176 & T & G & Missense SNV & NM_138420 & AHNAK2:NM_138420:exon7:C.A3444C:р..E1148D & 14932.33 & ID=COSM1368638;OCCURENCE=2(thyroid) & $0.765, \mathrm{~T}$ \\
\hline AHNAK2 & 14 & 105418391 & rs11625007 & c & T & Missense SNV & NM_138420 & AHNAK2:NM_138420:exon7:C:G3397A:P.VV11331 & 14432.33 & 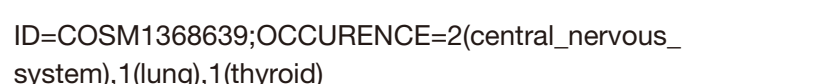 & 1.0,T \\
\hline AHNAK2 & 14 & 105419243 & NA & c & T & Missense SNV & NM_138420 & AHNAK2:NM_138420::Xxon7:C.G2545A:P.:G849S & 14932.33 & NA & \\
\hline muce & 3 & 195489009 & rs2246901 & c & A & Missense SNV & NM_004532,NM_188406,NM_138297 & 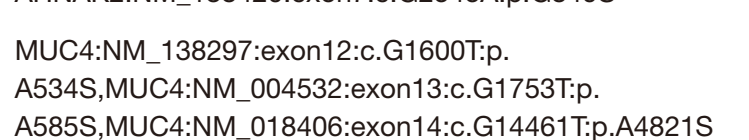 & 3029 & $\begin{array}{l}\text { IDCCOSM4157536,COSM4157534,COSM4157535;OCCCURE } \\
\text { NCE=2(thyroid) }\end{array}$ & $0.241, \mathrm{~T}$ \\
\hline MUC4 & 3 & 195489067 & rs2246980 & c & G & Synonymous SNV & NM_004532,NM_018406,NM_138297 & 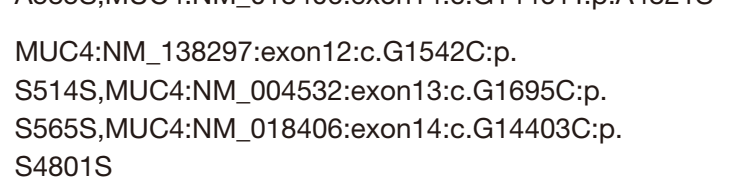 & 3929 & $\begin{array}{l}\text { ID=COSM1 1421868,COSM11421867,COSM1421 1869;OCCURE } \\
\text { NCE=1(large_intestine) }\end{array}$ & NA \\
\hline muc4 & 3 & 195510762 & rs2911273 & G & A & Synonymous SNV & NM_018406 & MUC4:NM_018406::xon22:C.C76889T:PV.V2563V & $3 \mathrm{q} 29$ & 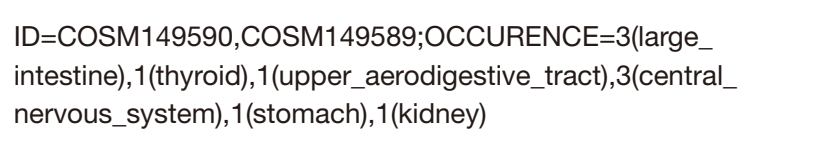 & NA \\
\hline MUC4 & 3 & 195512446 & rs550380149 & G & A & Missense SNV & NM_018406 & MUC4:NM_018406::xxn22:CCC6005T:P.P2002L & 3q29 & NA & \\
\hline muc4 & 3 & 195512597 & rs66799339 & a & A & Missense SNV & NM_018406 & MUC4:NM__18406::xxn22:C.C5854T:P.P1952S & 3229 & 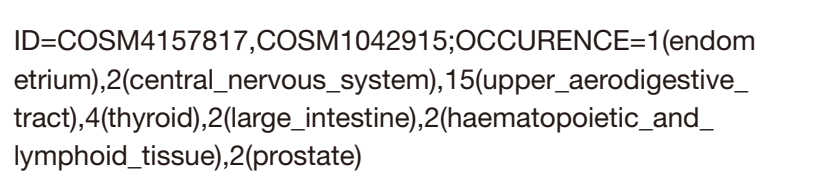 & $0.14, \mathrm{~T}$ \\
\hline Muc4 & 3 & 195513063 & NA & G & A & Synonymous SNV & NM_.018406 & MUC4:NM_018400::xxn22:C.С5388T:P.T1796T & 3929 & NA & NA \\
\hline MUC4 & 3 & 195513345 & rs 571321841 & A & c & Synonymous SNV & NM_0184066 & MUC4:NM_018400::exon2::T5106G:P.,V1702V & 3929 & 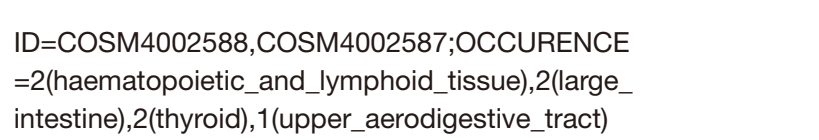 & NA \\
\hline muc4 & 3 & 195515008 & rs201451131 & c & a & Missense SNV & NM_018406 & 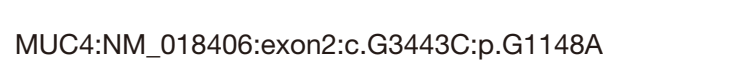 & $3 \mathrm{q} 29$ & 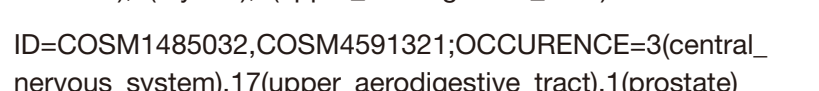 & 1.0,T \\
\hline MUC4 & 3 & 195517321 & rs 1104760 & G & A & Missense SNV & NM_018406 & MUC4:NM_018406::Xxon2:C.C1130T:P.T3771 & 3929 & 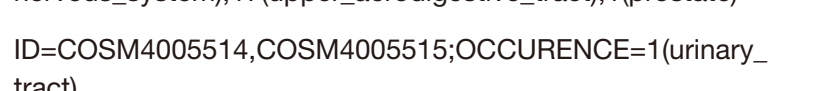 & 1.0,T \\
\hline SULtTAT & 16 & 28617507 & rs412788160 & c & T & Synonymous SNV & $\begin{array}{l}\text { NM_O01055,NM_177529,NM_177530, } \\
\text { NM__77534,NM_177536 }\end{array}$ & 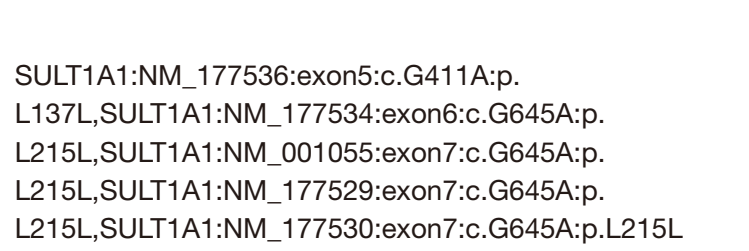 & 16p11.2. & 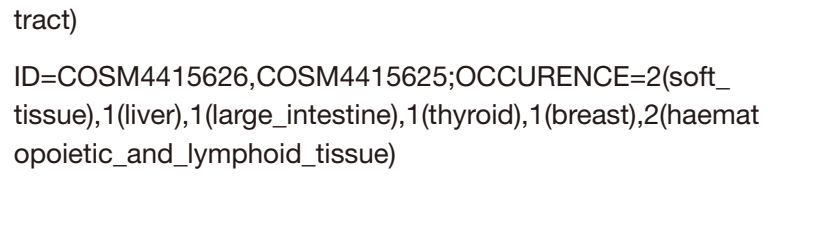 & NA \\
\hline SULtTAT & 16 & 2867572 & rsi171724912 & a & A & Unknown & 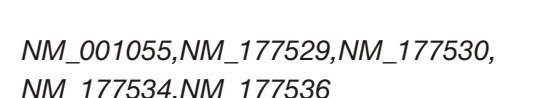 & unknown & $16 \mathrm{p} 11.2$ & ID=COSN267282843;0CCURENCE=1(breast) & NA \\
\hline SULtTAT & 16 & 28618237 & rs9282862 & T & c & Unknown & $\begin{array}{l}\text { NM_0101055,NN_177529,NM_177530, } \\
\text { NM__77534,NM_177536 }\end{array}$ & unknown & $16 \mathrm{pp} 1.2$ & 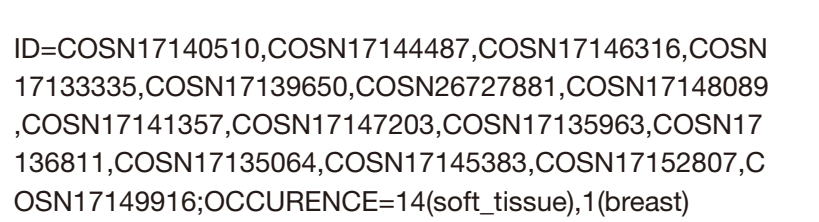 & NA \\
\hline SULtTAT & 16 & 28618446 & rs2925623 & T & c & Unknown & $\begin{array}{l}\text { NM_o01055,NM_177529,NM_177550, } \\
\text { NM_177534,NM_ } 777536\end{array}$ & unknown & 16011.2 & 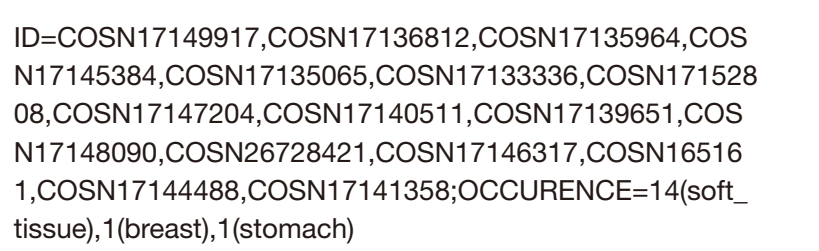 & NA \\
\hline SULtTAT & 16 & 28618469 & rs3764879888 & A & T & Unknown & $\begin{array}{l}\text { NM_O01055,NM_177529,NM_177550, } \\
\text { NM_177534,NM_177536 }\end{array}$ & unknown & $16 \mathrm{p} 11.2$ & 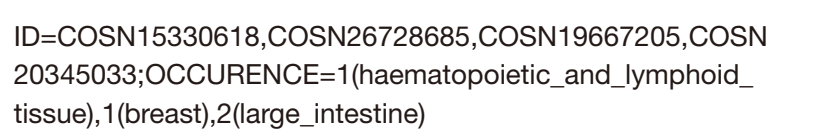 & NA \\
\hline SULtiat & 16 & 28618636 & rs30200804 & A & a & Unknown & 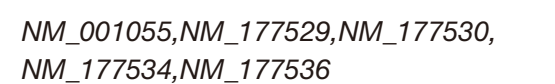 & unknown & $16 \mathrm{p} 11.2$ & 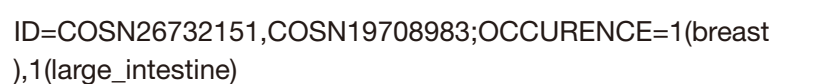 & NA \\
\hline SULtAAT & 16 & 28619911 & rst126447 & T & c & Synonymous SNV & $\begin{array}{l}\text { NM_001055,NM_177529,NM_177530, } \\
\text { NM_177534 }\end{array}$ & 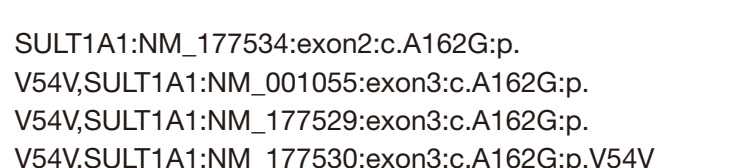 & 16011.2 & 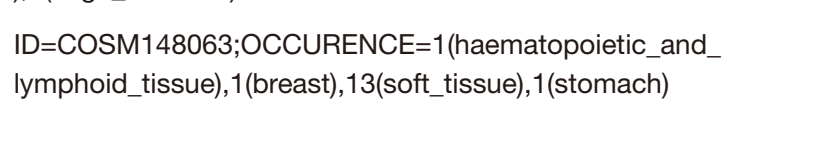 & NA \\
\hline SULtAAT & 16 & 28619920 & rs1126446 & A & a & Synonymous SNV & $\begin{array}{l}\text { NM_001055,NM_177529,NM_177530, } \\
\text { NM_177534 }\end{array}$ & 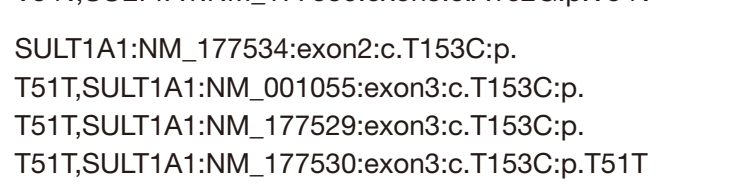 & $16 \mathrm{p} 11.2$ & 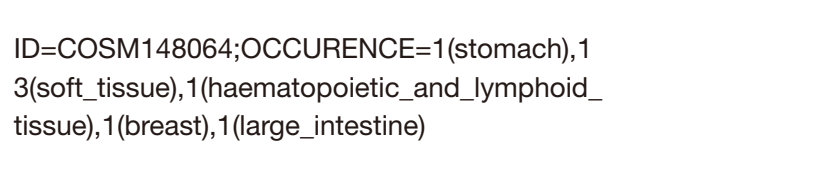 & NA \\
\hline SULtTAT & 16 & 28620120 & rs 834513973 & c & T & Synonymous SNV & $\begin{array}{l}\text { NM_o01055,NM_177529,NM_177530, } \\
\text { NM_-77554 }\end{array}$ & 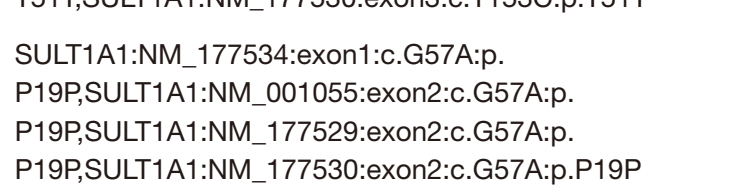 & $16 \mathrm{pp} 1.2$ & 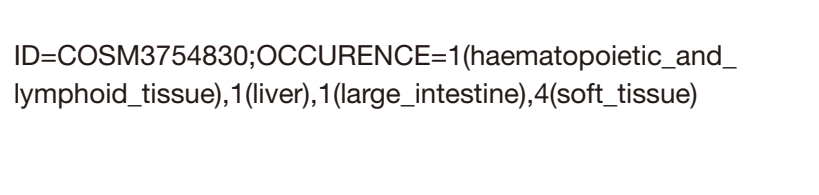 & NA \\
\hline NBPF1 & 1 & 16890417 & NA & T & G & Unknown & NM_017940 & unknown & 1p36.13 & NA & NA \\
\hline NBPF1 & 1 & 16890441 & rs3863779 & c & T & Unknown & NM_017940 & UNKNOWN & 1p36.13 & 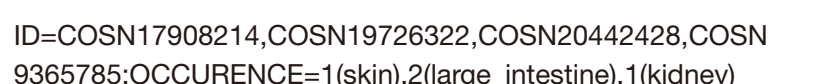 & NA \\
\hline NBPF1 & 1 & 16890484 & rst2117084 & a & c & Unknown & NM_017940 & UNKNOWN & $1 \mathrm{p36.13}$ & 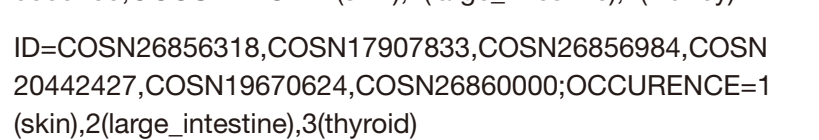 & NA \\
\hline NBPF1 & 1 & 16892282 & rs202078823 & c & A & Unknown & NM_017940 & unkNown & 1p36.13 & 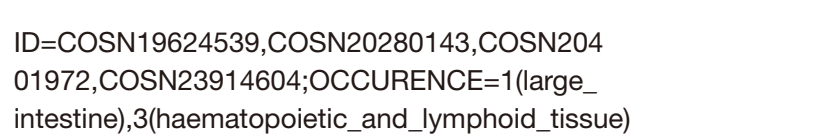 & NA \\
\hline NBPF1 & 1 & 16893598 & rs927016856 & G & A & Unknown & NM_017940 & unknown & $1 \mathrm{p36.13}$ & ID=COSN1091962;:OCCURENCE=1(liver) & NA \\
\hline NBPF1 & 1 & 16903026 & rs3897291 & c & A & Unknown & NM_017940 & unknown & 1p36.13 & 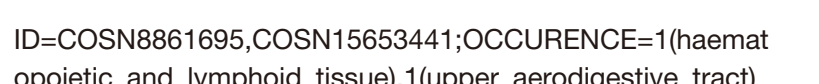 & NA \\
\hline NBPF1 & 1 & 16907164 & rs201839566 & c & $\mathrm{T}$ & Unknown & NM_017940 & unknown & $1 \mathrm{p36} .13$ & 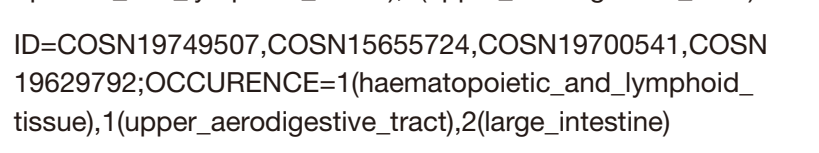 & NA \\
\hline NBPF1 & 1 & 16918411 & rs199725761 & T & c & Unknown & NM_017940 & & 1936.13 & ID=COSN19740211;OCCURENCE=1(large_intestine) & NA \\
\hline ABO & 9 & & rs8176749 & c & T & Unknown & NM_020469 & & 9934.2 & NA & \\
\hline ABO & 9 & 1315 & rs8176747 & c & G & Unknown & NM_020469 & & 3.2 & $\mathrm{ID}=\cos$ & NA \\
\hline$A B O$ & 9 & & rs8176746 & G & T & Unknown & NM_020469 & & 4.2 & NA & NA \\
\hline ABO & 9 & & rs8176743 & c & T & Unknown & NM_020469 & & 9934.2 & NA & NA \\
\hline ABO & 9 & & rs8176741 & G & A & Unknown & NM_020469 & Nown & 9934.2 & NA & NA \\
\hline ABO & 9 & & rs78559899 & G & c & Unknown & NM_o20469 & UNKNOWN & 9934.2 & NA & NA \\
\hline ABO & 9 & & rs8176722 & c & A & Unknown & NM_020469 & nown & 9934.2 & NA & NA \\
\hline MSTIL & 1 & & rs749078768 & A & a & Missense SNV & NM_001271733 & MST1L:NM__001271733:exon14:C.T1835C:P.1612T & $1 \mathrm{p36.13}$ & NA & NA \\
\hline MSTIL & 1 & 17084152 & rs202123117 & c & T & Missense SNV & NM_-0012717733 & MST1L:NM_001271733:exon14:CG.G1769AA:P.G5900 & 1036.13 & 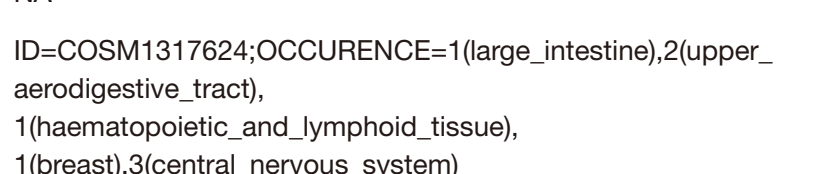 & NA \\
\hline MSTLL & 1 & 17085046 & rs200838083 & c & a & Missense SNV & NM_001271733 & MST1L:NM__001271733:exon11:CGG1429C:P.D477H & $1 \mathrm{p36.13}$ & 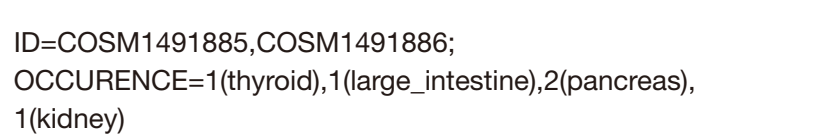 & NA \\
\hline MSTLL & 1 & 17086236 & rs661769737 & G & c & Unknown & NM_-001271733 & unknown & $1 \mathrm{p36} .13$ & 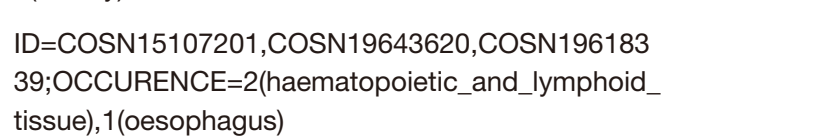 & NA \\
\hline MSTLL & 1 & 17086273 & NA & T & A & Unknown & NM_001271733 & unknown & $1 \mathrm{p36} .13$ & 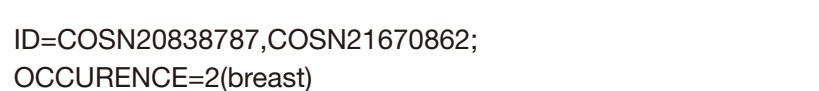 & NA \\
\hline MSTIL & 1 & 17087432 & rs2446554 & a & T & Unknown & NM_O01277 & unknown & $1 \mathrm{p36} .13$ & ID=COSN19672857;0CCCURENCE=1(arge_intestine) & NA \\
\hline ZNF718 & 4 & 53310 & rs761021532 & c & T & Unknown & 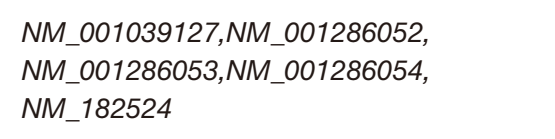 & unknown & 4p16.3 & NA & \\
\hline ZNF718 & 4 & 53360 & rs758588144 & c & a & Unknown & 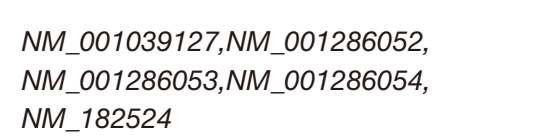 & unknown & 4p16.3 & ID=COSN26958394;OCCURENCE=1(lung) & NA \\
\hline ZNF718 & 4 & 59350 & rs 1045387254 & A & a & Missense SNV & $\begin{array}{l}\text { NM__001039127,NM__001286052, } \\
\text { NM } 182524\end{array}$ & ZNFF71:NM__0010399127:exon2:AA31G:P.111V & 4p16.3 & 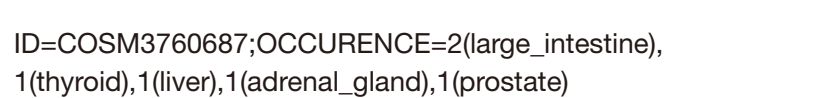 & $0.15, \mathrm{~T}$ \\
\hline ZNF718 & 4 & 59469 & rs6834940 & A & a & Unknown & 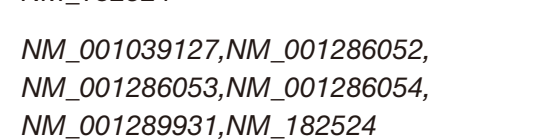 & unknown & 4p16.3 & $\begin{array}{l}\text { ST19766576, } \\
\text { Narge__ntestine) }\end{array}$ & NA \\
\hline ZNF718 & 4 & 60210 & rs3908749 & G & A & Unknown & 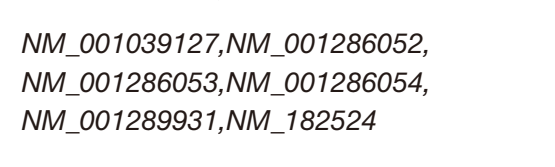 & unknown & 4p16.3 & 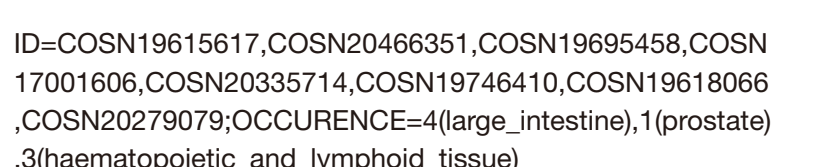 & NA \\
\hline ZNF718 & 4 & 67801 & rs777175674 & c & T & Unknown & 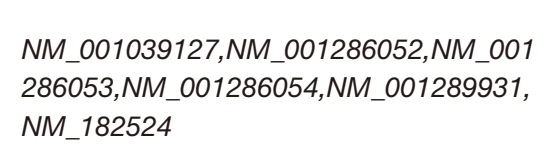 & unknown & 4p16.3 & $\begin{array}{l}\text { ID=COSN } 19699753, \text {,COSN19751413; } \\
\text { OCCURENCE=2(arge_intestine) }\end{array}$ & NA \\
\hline MUC12 & 7 & 35014 & r55423220663 & c & T & Synonymous SNV & NM_001164462 & & $7 \mathrm{q} 22.1$ & & \\
\hline MUC12 & 7 & 35205 & rs4729631 & c & T & Missense SR & NM_001164462 & MUC12:NM__001164462:8xon2:C.C1361TT:P.A45 & $7 \mathrm{q} 22.1$ & $\begin{array}{l}\text { ID=COSM4004183,COSM40044182; } \\
\text { OCCURENCE=2haemanatopoiticand_Iymphoid_tissue) }\end{array}$ & \\
\hline muc12 & 7 & 100637193 & rs10247974 & c & A & Missense SNV & NM_001164462 & MUC12:NM_001164462::XXn2::C.C3349A:P.P.P1117T & 7922.1 & 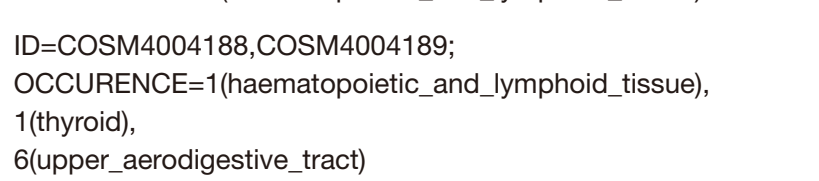 & $0.0, \mathrm{D}$ \\
\hline muc12 & 7 & 10063828 & rs569914801 & c & T & Missense SNV & NM_001164462 & MUC12:NM_001164462::Xxon2:C.C4984TT.P.P1662S & $7 \mathrm{q} 22.1$ & 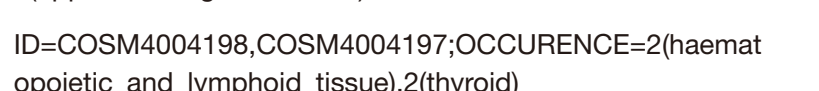 & $0.237, \mathrm{~T}$ \\
\hline MUC12 & 7 & 100646938 & rs199993063 & c & G & Missense SNV & & & $7 \mathrm{q} 22.1$ & NA & $0.0, \mathrm{D}$ \\
\hline Muc12 & 7 & 100647073 & rs 140284525 & $\mathrm{c}$ & T & Missense SNV & NM_001164462 & MUC12:NM_001164462:exon2:C.CC13229T:P.T4410M & $7 \mathrm{q} 22.1$ & 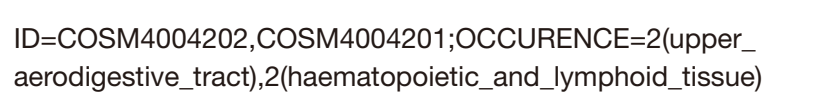 & $0.003, \mathrm{D}$ \\
\hline MUC16 & 19 & 9024994 & rs6763121215 & c & T & Missense SNV & NM_024690 & MUC16:NM_024690::8xon16:c.G336868A:p.E12290K & 19p13.2 & 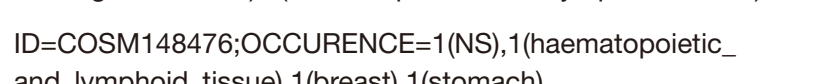 & $0.044, \mathrm{~L}$ \\
\hline мuc16 & 19 & 9059159 & rs12462651 & T & a & Missense SNV & NM_o24690 & MUC16:NM__224690::xon3:C.A28287C:P..K9429N & 19p13.2 & 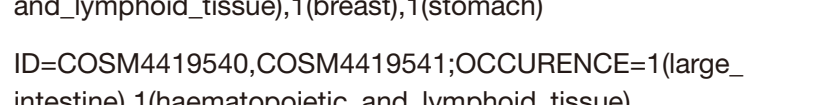 & $0.013, \mathrm{~L}$ \\
\hline MUC16 & 19 & 9000056 & rs2216663 & c & T & Synonymous SNV & NM_024690 & MUC16:NM__244600:exon3:C.G667900A:p.E.E930E & 19p13.2. & 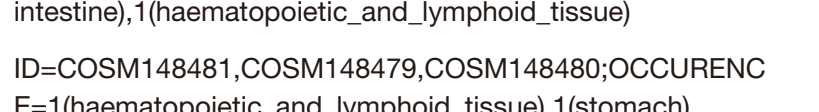 & \\
\hline & 19 & 9061454 & & c & A & Missense SNV & & & & macn) & \\
\hline $\begin{array}{l}\text { Muc16 } \\
\text { muc16 }\end{array}$ & $\begin{array}{l}19 \\
19\end{array}$ & $\begin{array}{l}9062847 \\
9080462\end{array}$ & $\begin{array}{l}\text { rs79203775 } \\
\text { rs2547065 }\end{array}$ & $\begin{array}{l}\text { A } \\
\text { C }\end{array}$ & $G_{G}^{G}$ & $\begin{array}{l}\text { Missense SNV } \\
\text { Missense SNV }\end{array}$ & NM_O24690 & 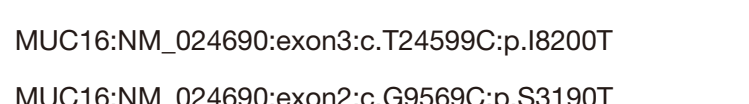 & $\begin{array}{l}19 p 13.2 \\
190132\end{array}$ & NA & $\begin{array}{l}0.003, \mathrm{D} \\
0.0, \mathrm{~T}\end{array}$ \\
\hline
\end{tabular}

